\title{
Extrapulmonary Pneumocystis Jirovecii Presenting as Fever and Lymphadenopathies. Case Report and Literature Review
}

\author{
Marcelo V Radisic1*, Andrea Landucci1*, Boris Elsner², Laura \\ Linares $^{3}$, Melina Ferreiro ${ }^{3}$, Roberto 0 Froment $^{3}$ and Ana Terusi ${ }^{4}$ \\ 1'Internal Medicine Department, Sanatorio Finochietto. Av. Córdoba 2678. C1187AAN. \\ Ciudad Autónoma de Buenos Aires, Argentina
}

\section{Review Article \\ Volume 2 Issue 2}

Received Date: June 25, 2018

Published Date: June 29, 2018

${ }^{2}$ Centro de Patología. Peña 2967 - CP:1425AVI. Ciudad Autónoma de Buenos Aires, Argentina

3Internal Medicine Department, Sanatorio Finochietto. Av. Córdoba 2678. C1187AAN. Ciudad Autónoma de Buenos Aires, Argentina

${ }^{4}$ Infectious Disease Consultant. Internal Medicine Department, Sanatorio Finochietto. Av. Córdoba 2678. C1187AAN. Ciudad Autónoma de Buenos Aires, Argentina

*Corresponding author: Marcelo V Radisic, Internal Medicine Department, Sanatorio Finochietto. Av. Córdoba 2678, C1187AAN, Ciudad Autónoma de Buenos Aires, Argentina, Tel: +54-9-11-3374-9585; Email: marceloradisic@gmail.com Andrea Landucci, Internal Medicine Department, Sanatorio Finochietto. Av. Córdoba 2678, C1187AAN, Ciudad Autónoma de Buenos Aires, Argentina, Tel: +54-9-11-5519-6677; Email: andrealanducci@yahoo.com.ar

\section{Abstract}

Pneumocystosis is one of the most common opportunistic infection occurring in HIV infected patients. The usual clinical presentation of Pneumocystis infection is pulmonary. Extrapulmonary Pneumocystis (EP) infections are uncommon, and have generally been observed in patients with advanced HIV disease, many times under second line prophylaxis with aerosolized pentamidine. Because of its low frequency, its low index of suspicion and a variety of clinical presentations, extrapulmonary forms of $P$. jirovecii infections are usually difficult to diagnose. We report a case of extrapulmonary pneumocystosis as a form of presentation of Acquired Immunodeficiency Syndrome (AIDS) presenting with fever and enlarged intra-abdominal lymph nodes, and review all published cases of extrapulmonary pneumocystosis from 1997 to 2017.

Keywords: Pneumocystis Jirovecii; AIDS; HIV; Extrapulmonary Infection; Lymphadenopathy

\section{Introduction}

Symptomatic infection caused by Pneumocystis Jirovecii is an opportunistic infection that occurs almost exclusively in immunocompromised hosts. Due to the microorganism's low intrinsic virulence, infection in immunocompetent host is usually subclinical. Pneumocystosis is the most common opportunistic 
infection occurring in HIV infected patients infected in the USA [1]. The usual clinical presentation of Pneumocystis infection is pulmonary, characterized by an insidious onset, with fever, dyspnea, dry cough and diffuse interstitial pulmonary infiltrates. Extrapulmonary Pneumocystis (EP) infections are uncommon, and have generally been observed in patients with advanced HIV disease, many times under second line prophylaxis with aerosolized pentamidine.

Extrapulmonary infections may be present with no simultaneous pulmonary involvement, and may be limited to a single anatomical site. However, when multiple noncontiguous sites are involved, pulmonary disease is usually also present. Due to its low frequency, its low index of suspicion and a variety of clinical presentations, extrapulmonary forms of $P$. jirovecii infections are usually difficult to diagnose.

\section{Case Report}

A 30-year-old male was admitted (June 16th, 2015) because of fever starting 2 months before, with daily fever spikes ( $>380 \mathrm{C})$. At the beginning of his disease, the patient presented acute diarrhea, which resolved with antibiotic treatment (the patient was unable to recall the specific antibiotic). Before his admission, he consulted another healthcare facility, and tricytopenia and abdominal lymphadenopathies were observed. He was derived to our institution. On physical examination he presented inguinal lymphadenopathies of hard, rubbery consistence, and a non-pruritic, generalized rash (mainly in the trunk and upper limbs), which blanched under glass pressure. The patient related the rash to the recent administration of dipirone, and the rash disappeared without specific treatment after $48 \mathrm{hrs}$.

The laboratory test results showed: hematocrit 31\%, hemoglobin $10.7 \mathrm{~g} / \mathrm{dL}$, white blood cell count $2800 / \mathrm{mm}^{3}$ (71\% neutrophils, 6\% lymphocytes), platelet count $140.000 / \mathrm{mm}^{3}$, LDH $1050 \mathrm{UI} / \mathrm{L}$, serum ferritin 29,736 $\mathrm{ng} / \mathrm{mL}$. An HIV-ELISA tested positive (with further confirmation). Kidney and liver function test were normal, as well as the coagulogram. As part of the initial evaluation, blood and urine cultures were obtained. All cultures were negative. Chest $\mathrm{x}$-ray, electrocardiogram and Doppler echocardiogram showed no abnormalities.

Computed tomography (CT) of chest, abdomen and pelvis with intravenous contrast were obtained (Figure 1).

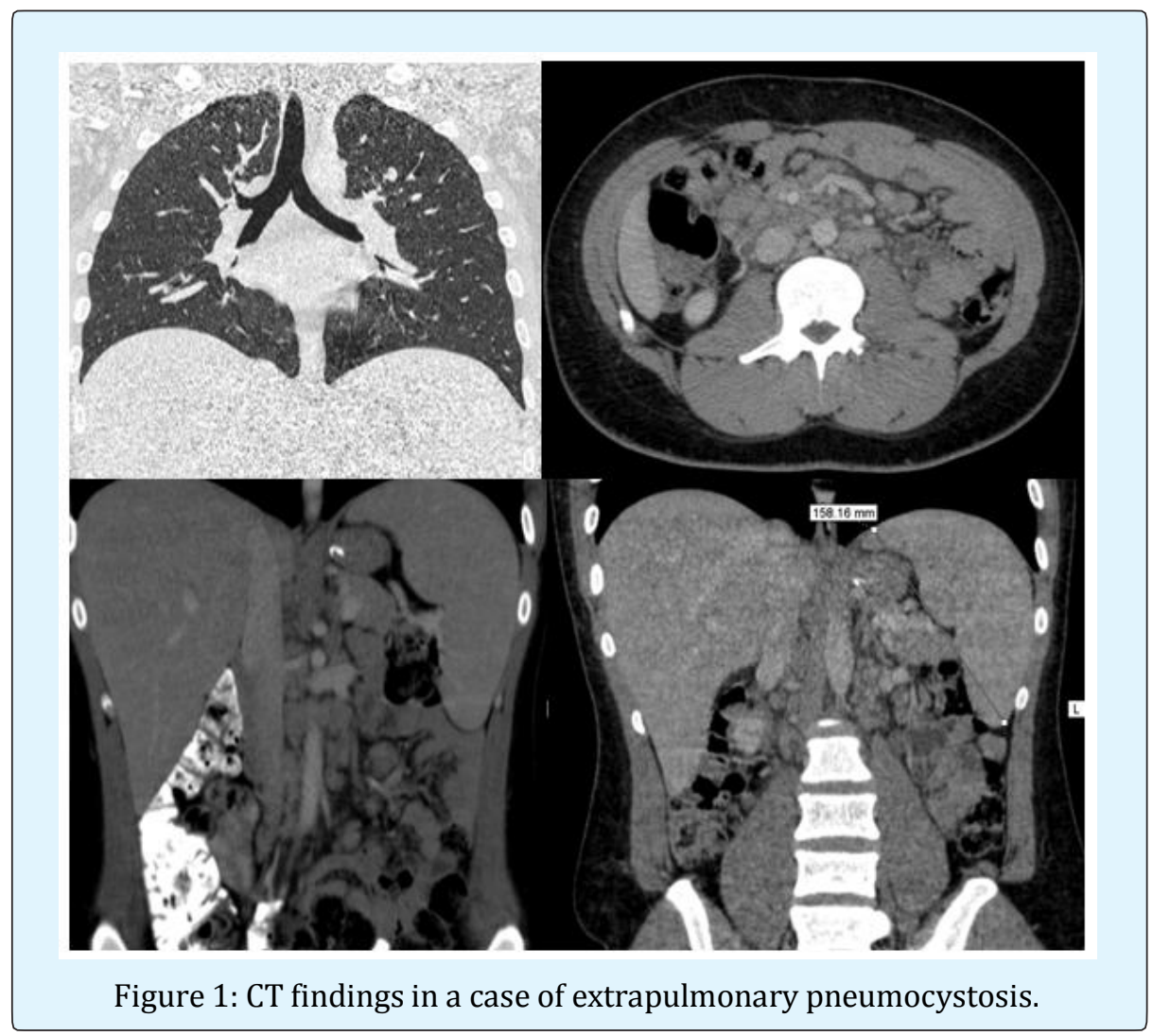

Marcelo V Radisic, et al. Extrapulmonary Pneumocystis Jirovecii Presenting as Fever and Lymphadenopathies. Case Report and Literature Review. J Inf Dis Trav Med 2018, 2(2): 000113. 
Computed tomography (CT) of thorax, abdomen and pelvis with intravenous contrast were obtained (Figure 1), showing spleen and liver enlargement with homogeneous density. Enlarged lymph nodes were noted at the retroperitoneum, para-aortic space, portacaval space, retro pancreatic space and at the mesentery root, with extension up to the iliac arteries bifurcation. Thorax CT showed no abnormalities. showing spleen and liver enlargement with homogeneous density. Enlarged lymph nodes were noted in the retroperitoneum, para-aortic space, portacaval space, retropancreatic space and at the mesentery root, with extension up to the iliac arteries bifurcation. Chest CT showed no abnormalities. Mycobacterial and fungal blood cultures were negative. Other serological and microbiological tests are presented in Table 1.

A bone marrow biopsy and a laparoscopic biopsy of retroperitoneal lymph nodes were obtained, and sent for pathological examination and flow cytometry analysis. Samples for mycobacterial, fungal and usual microorganism cultures were also collected. Antimicrobial prophylaxis with azithromycin and trimethoprim- sulfamethoxazole (TMP-SMX) was started. Because a lymphoproliferative process was suspected, prednisone $40 \mathrm{mg}$ /day was started. On June 23rd, results were received for absolute CD4+ count $\left(35 / \mathrm{mm}^{3}\right)$ and HIV viral load (298,000 copies /mL).

\begin{tabular}{|c|c|}
\hline EBV-IgG & Positive \\
\hline EBV-IgM & Negative \\
\hline EBNA & Negative \\
\hline EBV-EA & Negative \\
\hline Viral load EBV & Non- detectable. \\
\hline CMV-IgG & Positive \\
\hline CMV-IgM & Negative \\
\hline Viral load CMV & Non- detectable. \\
\hline Toxoplasmosis serology & Non- reactive \\
\hline Chagas disease serology & Non- reactive \\
\hline PVB19 IgG & Negative \\
\hline VDRL & Negative \\
\hline T. pallidum-HA assay & Negative. \\
\hline FTAbs & $+1 / 4$ positive. \\
\hline C. neoformans antigenemia & Negative. \\
\hline H. Capsulatum antibodies & Negative. \\
\hline HBsAg and antibodies to HCV & Negative. \\
\hline
\end{tabular}

Table 1: Microbiological and serological tests results. EBV: Epstein Barr virus; EBNA: Epstein Barr Nuclear Antigen; EA: early antigen; CMV: cytomegalovirus; PV: Parvovirus.

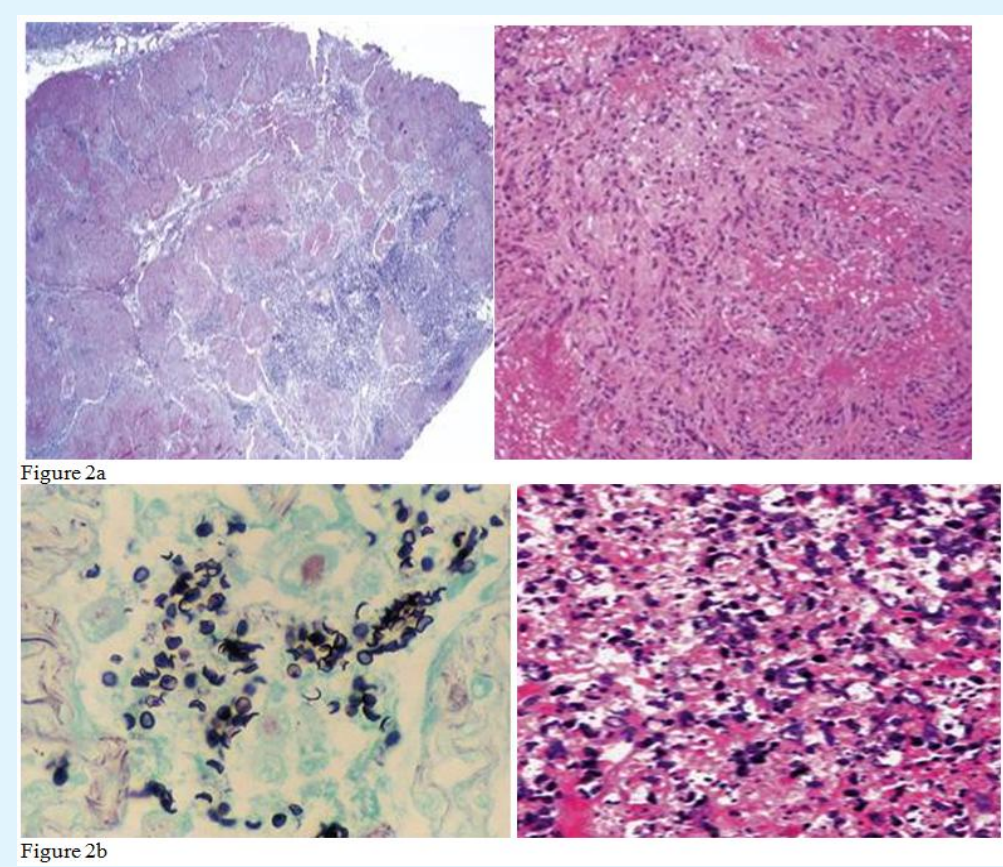

Figure 2(a,b): PAS and Grocott stains shows numerous elements, 5-8 microns large, mainly extracelullar, intensively PAS-positive, with a rounded or oval morphology and a PAS positive-central area, resembling "crushed ping pong balls", a morphology corresponding to P. jirovecii cysts. 
Antiretroviral drugs (tenofovir, lamivudine and raltegravir) were requested to start treatment. Bone marrow and lymph node cultures yielded no microorganism growth.

Flow cytometry analysis and pathology of the bone marrow showed a marked inversion of CD4 to CD8 ratio, with no phenotypic anomalies, no evidence of lymphoproliferative disease, and with marked reactive changes.

On July 1st, the initial report on the retroperitoneal lymph node informed atypical lymphoid hyperplasia, "probably related to viral infection". Later, special stains(PAS and Grocott) revealed numerous elements, 5-8 microns large, mainly extracellular, intensely PASpositive, with a rounded or oval morphology and a PAS positive-central area, resembling "crushed ping pong balls", a morphology corresponding to $P$. jirovecii cysts (Figure 2). Immunofluorescent staining with specific monoclonal antibodies confirmed the diagnosis.

Treatment with intravenous TMP-SMX $(15 \mathrm{mg} / \mathrm{kg} /$ day trimethoprim) was then started. The patient's clinical condition rapidly improved, becoming afebrile on day 6 after starting treatment. On day 13, TMP-SMX was changed to oral administration, and the patient was discharged. He completed 21 days of TMP-SMX. The patient fully recovered from his infection, but he was lost to follow-up when he returned to his home country (Brazil) 2 months after discharge.

\section{Discussion}

Pneumocystis Jirovecii is one of the most important opportunistic pathogen affecting AIDS patients. Incidence of $P$. jirovecii infection (which before the availability of highly active antiretroviral treatment, (HAART), affected up to $75 \%$ of HIV infected patients) is around 3,9/1000 year-patient [2], and it is still the most common opportunistic infection in this patient group, especially in those with low CD4 count or in patients unaware of their HIV infection.

Although it has been formerly classified as a protozoa, $P$. jirovecii is a yeast-like fungus, classified as such on the basis of genomic sequences of its ribosomal RNA, its wall chemical composition, and the structure of key enzymes [3]. However, Pneumocystisis an atypical fungus that does not grow in culture media.

Human Pneumocystis is an anthropozoonosis, being humans the only reservoir and host. Transmission is primarily airborne (usually from other infected persons, and in certain occasions, possibly from environmental reservoirs) [4]. Colonization with P. jirovecii occurs in the early stages of life, but in the presence of a normal immunologic response, the infection is contained, although not eradicated. Infection persists in a dormant state, with the potential of progression to clinical disease if there is a derangement of the host's immunological response. Rarely, a recently acquired infection may progress to pulmonary and eventually extrapulmonary clinical disease. From the lung, the microorganism may spread systemically either directly or by lymphatic or hematogenous pathways, although dissemination is distinctly unusual.

Pulmonary pneumocystosis is usually an interstitial pneumonia with symptoms as fever, cough and dyspnea. Radiological appearance is customarily a diffuse reticular or finely granular interstitial infiltrate. On CT, bilateral patchy "ground glass" asymmetric infiltrates, with upper lobes predominance is usually observed [5]. Occasionally, lung pneumocystosis may occur in the absence of abnormal CT findings.

Although pulmonary pneumocystosis is the most prevalent serious lung infection in HIV infected patients, extrapulmonary pneumocystosis is uncommon.

P. jirovecii has the potential to disseminate from the lung to other organs, producing infection in almost any anatomic site. Endothelial damage due to Kaposi's sarcoma, cytomegalovirus infection, or recurrent $P$. jirovecii infection may facilitate dissemination from the lung.

Before HIV epidemic, extrapulmonary pneumocystosis was extremely rare (only 16 cases reported from 1954 to 1996, mainly post-mortem studies). HIV epidemic was accompanied by an increase in Pneumocystis cases, and by an increase in extrapulmonary pneumocystosis reports. The true incidence of extrapulmonary pneumocystosis is unknown, although it has been estimated in $0.06-2,5 \%$ [6-8].

Pulmonary infection and extrapulmonary infection have been considered to be caused by different genetic variants of $P$. jirovecii, variants that may potentially differ in their susceptibility to antimicrobial treatments [9].

Extrapulmonary pneumocystosis has been observed in HIV infected patients receiving aerosolized pentamidine prophylaxis. The distribution of this drug within the lung, when administered this way, is not uniform [10] and it 
has no systemic effects. Therefore, it may be insufficient to prevent systemic dissemination and to suppress $P$. jirovecii out of the lung, which explains its association as a risk factor for extrapulmonary pneumocystosis $[11,6]$.

Other risk factors observed are advanced stages of HIV infection with no prophylaxis -in these patients, extrapulmonary pneumocystosis without simultaneous lung disease may be observed-, low CD4 count,and ahistory of lung infections.

Extrapulmonary pneumocystosis has been described in eyes (usually affecting the choroidal layer), ear, thyroid, spleen and many other location [12].

Cases published as of 1997 have been thoroughly reviewed by $\mathrm{Ng}$, et al. The epidemiology of extrapulmonary pneumocystosis may have changed after that, as the availability and tolerability of HAART increased along the years. We therefore searched for cases (either in English or Spanish, in PubMed and Scielo databases) not included in $\mathrm{Ng}$ review, published from 1997 to 2017.

Lymph nodes are the most common site for extrapulmonary $P$. jirovecii infection in HIV positive patients. Among the 90 cases (published between 1982 and 1996) reviewed by $\mathrm{Ng}$, et al. 19 (21\%) had lymph node involvement13.

In a review of 233 autopsy findings (HIV positive patients died between from 1985 to 1996), pulmonary pneumocystosis was observed in 56 patients. Within this group, extrapulmonary involvement was observed in 7 (13\%), six of them with lymph node disease due to $P$. jirovecii [13].Intrathoracic adenopathies have been documented in $18 \%$ of chest CTs in patients with pulmonary pneumocystosis, although as a rule, lymph node involvement is unapparent in usual chest $\mathrm{x}$ - ray [14].

It is possible that the clinical behavior of P. jirovecii may have varied according with the increased availability of better antiretroviral treatments. Initial observations include patients from a time in which many of the currently used antiretroviral drugs were not still available (by 1994, the only approved drugs were zidovudine, didanosine, zalcitabine and stavudine). In the reviewed literature (published in English and Spanish, from 1997 to 2017), among 34 cases of extrapulmonary pneumocystosis, 10 cases presented documented or potential lymph node involvement caused by P.jirovecii: In 6 post-mortem cases, a detailed description was not provided [13]. The four remaining cases comprised one patient with pulmonary pneumocystosis who developed hilar adenopathies and pleural masses while on dapsone prophylaxis (not biopsied, but resolved with antiPneumocystis treatment) [15]; a 7-year-old boy with generalized lymphadenopathies, in whom fine needle aspiration cytology allowed diagnosis [16]; one patient with mediastinal, cervical, and left supraclavicular lymphadenopathies (surgical biopsy positive for Pneumocystis) [17] and one patient with simultaneous documented involvement of lung and colon, with potential infection of mesenteric and retroperitoneal lymph nodes (not biopsied) [18].

Relevant clinical data from extrapulmonary Pneumocystis cases published from 1997 to 2017 is summarized in Table 2 [13,15-32].

Additionally, one 50-year-old male patient was reported in an Italian journal in 1998. He was diagnosed with AIDS 8 months before, treated with zidovudine and didanosine, with an absolute CD4 count $6 / \mathrm{mm} 3$. He developed a severe respiratory pneumocystosis (in spite of being on aerosolized pentamidine prophylaxis) and was treated with pirimethamine and clindamycin. He died on day 14 after diagnosis. On autopsy, a massive pulmonary embolism was observed. Grocott stain allowed to document persistence of pulmonary infection and hilar and mediastinal lymphatic dissemination of Pneumocystis [33].

Because Pneumocystis cannot be readily cultured in the routine laboratory, the gold standard for diagnosis is the direct visualization of the organisms. Extrapulmonary $P$. jirovecii infection is diagnosed by the observation of the typical cyst in formalin-fixed tissue. It should be noted that $P$. jirovecii is usually very difficult to identify with hematoxilin-eosin or Papanicolaou stains, and they stain faint and variably. Grocott stain (silver methenamine) allow the organism to be seen mainly as spherical cysts, with variations in shape. Occasionally, small interior structures can be observed in the form of commas, dots, or rings. Differential diagnosis include organisms similar to $P$. jirovecii, such as Histoplasma capsulatum, capsuledeficient Cryptococcus, Penicillium marneffei, endospores of Coccidioides spp and Candida species, but these spores usually have a budding appearance with is absent in $P$. jirovecii cysts [16,34]. Direct immunofixation or PCR techniques can be used for diagnosis in difficult cases.

Sometimes, (as when disease is localized in the eye) diagnosis is based on typical appearance of lesions and response to treatment. Prognosis is better for localized 


\section{Journal of Infectious Diseases \& Travel Medicine}

forms in eye or ear than for disseminated forms affecting non-contiguous sites [12].

The differential diagnosis of lymphadenopathies in patients with HIV infection includes HIV associatedlymphadenopathy, neoplastic diseases such as Kaposi sarcoma or lymphoproliferative diseases, and a variety of infectious etiologies such a mycobacterial infection (tuberculous and non-tuberculous) and fungal infections. Lymph node disease is a frequent diagnostic challenge for physicians who treat HIV infected patients, as up to $25 \%$ of HIV infected patients experience some form of lymph node involvement, at any point of the clinical progression of the disease, from early stages to advanced HIV infection. Our observation emphasizes that, although highly uncommon, extrapulmonary pneumocystosis may be considered in the differential diagnosis of lymph node disease.

Treatment of extrapulmonary pneumocystosis follows the same principles of pulmonary Pneumocystis treatment. Trimethoprim-sulfamethoxazole (TMP-SMX) remains the drug of choice for all forms of Pneumocystis infection. The recommended daily dose is TMP $15-20$ $\mathrm{mg} / \mathrm{kg}$ plus SMX $75-100 \mathrm{mg} / \mathrm{kg}$. The optimal treatment duration of extrapulmonary Pneumocystis has not been well studied, but it is generally recommended to treat HIV-infected patients for at least 21 days [35]. Second line therapy includes intravenous pentamidine, clindamycin plus primaquine, atovaquone, and dapsone plus TMP22.Caspofungin associated with clindamycin [36] or low doses SMX-TMP [37] may also be considered among the therapeutic alternatives.

\section{Conclusions}

The present case illustrates the importance of considering Pneumocystis in the differential diagnosis of lymphadenopathies in AIDS patients. Although uncommon, even in this era of highly active antiretroviral treatments, extrapulmonary $P$. jirovecii infections may still occur. Knowledge of the risk factors for extrapulmonary pneumocystosis (such as HIV infection advanced stage, with profound immunosuppression, or use of aerosolized pentamidine prophylaxis) and of the variety of extrapulmonary P.jirovecii clinical formsmay allow for timely diagnosis and appropriate management.

\section{References}

1. Morris A, Lundgren JD, Masur H, Walzer PD, Hanson DL, et al. (2004) Current epidemiology of

Marcelo V Radisic, et al. Extrapulmonary Pneumocystis Jirovecii Presenting as Fever and Lymphadenopathies. Case Report and Literature Review. J Inf Dis Trav Med 2018, 2(2): 000113.
Pneumocystis pneumonia. Emerg Infect Dis 10(10): 1713-1720.

2. Buchacz $\mathrm{K}$, Lau B, Jing $\mathrm{Y}$, Bosch $\mathrm{R}, \mathrm{Abraham} A \mathrm{~A}$, et al. (2016) North American AIDS Cohort Collaboration on Research and Design (NA-ACCORD) of IeDEA. Incidence of AIDS-Defining Opportunistic Infections in a Multicohort Analysis of HIV-infected Persons in the United States and Canada, 2000-2010. J Infect 214(6): 862-872.

3. Edman JC, Kovacs JA, Masur H, Santi DV, Elwood HJ, et al. (1988) Ribosomal RNA sequence shows Pneumocystis carinii to be a member of the fungi. Nature 334(6182): 519-522

4. Yiannakis EP, Boswell TC (2016) Systematic review of outbreaks of Pneumocystis Jirovecii pneumonia: evidence that $P$. jirovecii is a transmissible organism and the implications for healthcare infection control. J Hosp Infect 93(1): 1-8.

5. Kanne JP, Yandow DR, Meyer CA (2012) Pneumocystis jiroveci pneumonia: high-resolution CT findings in patients with and without HIV infection. AJR Am J 198(6): 555-561.

6. Coker RJ, Clark D, Claydon EL, Gompels M, Ainsworth JG, et al. (1991) Disseminated Pneumocystis carinii infection in AIDS. J Clin Pathol 44(10): 820-823.

7. Telzak EE, Cote RJ, Gold JWM, Campbell SW, Armstrong D (1990) Extrapulmonary Pneumocystis carinii infections. Rev Infect Dis 12(3): 380-386.

8. Raviglione MC (1990) Extrapulmonary pneumocystosis: the first 50 cases. Rev Infect Dis 12(6): 1127-1138.

9. Lecuit M, Livartowski J, Vons C, Goujard C, Lemaigre G, et al. (1994) Resistance to trimethoprimsulfamethoxazole and sensitivity to pentamidine therapy in an AIDS patient with hepatosplenic pneumocytosis. AIDS 8(10):1506-1507.

10. Ilowite JS, Baskin MI, Sheetz MS, Abd AG (1991) Delivered dose and regional distribution of aerosolized pentamidine using different delivery systems. Chest 99(5): 1139-1144.

11. Yoshida EM, Filipenko D, Phillips P, Montaner JS, Whittaker JS (1996) AIDS-related extrapulmonary Pneumocystis carinii infection presenting as a solitary rectal ulcer. Can J Gastroenterol 10: 401-404. 


\section{Journal of Infectious Diseases \& Travel Medicine}

12. Ng VL, Yajko DM, Hadley WK (1997) Extrapulmonary pneumocystosis. Clin Microbiol Rev 10(3): 401-418.

13. Afessa B, Green W, Chiao J, Frederick W (1998) Pulmonary complications of HIV infection: autopsy findings. Chest 113(5): 1225-1229.

14. Boiselle PM, Crans CA, Kaplan MA (1999) The changing face of Pneumocystis carinii pneumonia in AIDS patients. Am J Roentgenol 172(5): 1301-1309.

15. Sundar K, Rosado-Santos H, Reimer L, Murray K, Michael J (2001) Unusual presentation of thoracic Pneumocystis carinii infection in a patient with acquired immunodeficiency syndrome. Clin Infect Dis 32(3): 498-501

16. Anuradha, Sinha A (2007) Extrapulmonary Pneumocystis carinii infection in an AIDS patient: a case report. Acta Cytol 51(4): 599-601.

17. Kallen AJ, Wallace MR (1998) Atypical pneumocystis carinii infections in AIDS: Massive cervical lymphadenitis and fever of unknown origin. South Med J 91: 759-760

18. Valdebenito C, Bonacic M, Matamala J, Wolff M (2015) Extrapulmonary pneumocystosis: a case report. Rev Chilena Infectol 32(3): 344-349.

19. Karam MB, Mosadegh L (2014) Extrapulmonary Pneumocystis Jirovecii infection: A case report. Braz J Infect Dis 18 (6): 681-685.

20. Peña ZG, Byers HR, Lehmer LM, Smith CA (2013) Ragsdale BD. Mixed Pneumocystis and Cryptococcus cutaneous infection histologically mimicking xanthoma. Am J Dermatopathol 35(1): e6-10.

21. O'Neal CB, Ball SC (2008) Splenic pneumocystosis: an atypical presentation of extrapulmonary Pneumocystis infection. AIDS Read 18(10): 503-508.

22. Mahlakwane MS, Ramdial PK, Sing Y, Calonje E, Biyana S (2008) Otic pneumocystosis in acquired immune deficiency syndrome. Am J SurgPathol 32(7): 1038-1043.

23. Panos GZ, Karydis I, Velakoulis SE, Falagas ME (2007) Multi-skeletal Pneumocystis jiroveci (carinii) in an HIV-seropositive patient. Int J STD AIDS 18(2): 134137.

24. Praveen CV, Terry RM, Elmahallawy M, Horsfield C (2002) Pneumocystis carinii infection in bilateral aural polyps in a human immunodeficiency viruspositive patient. J Laryngol Otol 116(4): 288-290.

25. Burckhardt B, Sendi P, Pfluger D, Zimmerli W, Nüesch $\mathrm{R}$, et al. (1999) Rare AIDS-defining diseases in the Swiss HIV Cohort Study. Eur J Clin Microbiol Infect Dis 18(6): 399-402.

26. Patel SK, Philpott JM, McPartlin DW (1999) An unusual case of Pneumocystis carinii presenting as an aural mass. J Laryngol Otol 113(6): 555-557.

27. OlcozGonñi JL, Morán Blanco A, Bahamonde Carrasco A, Alonso A, Suárez D, et al. (1998) Intestinal obstruction and necrotizing thyroiditis secondary to Pneumocystis carinii infection in a seropositive hemophiliac patient seropositive for human immunodeficiency virus. Gastroenterol Hepatol 21(1): 13-15.

28. Kinchen $\mathrm{K}$, Kinchen $\mathrm{TH}$, Inglesby $\mathrm{T}$ (1998) Pneumocystis carinii infection of the small intestine. J Natl Med Assoc 90(10): 625-627.

29. Ruggli GM, Weber R, Messmer EP, Font RL, Moll C, et al. (1997) Pneumocystis carinii infection of the conjunctiva in a patient with acquired immune deficiency syndrome. Ophthalmology 104 (11): 18531856.

30. Bartlett JA, Hulette C (1997) Central nervous system pneumocystosis in a patient with AIDS. Clin Infect Dis 25(1): 82-85.

31. Villanueva JL, Cordero E, Caballero-Granado FJ, Regordan C, Becerril B, et al. (1997) Pneumocystis carinii meningo radiculitis in a patient with AIDS. Eur J ClinMicrobiol Infect Dis 16(12): 940-942.

32. Bundow DL, Aboulafia DM (1997) Skin involvement with Pneumocystis despite dapsone prophylaxis: a rare cause of skin nodules in a patient with AIDS. Am J Med Sci 313(3): 182-186.

33. Manfredi R, Salfi N, Pasquinelli G, Chiodo F (1998) [Lymph node dissemination of a serious Pneumocystis carinii respiratory infection in AIDS: histopathological and ultrastructural aspects]. Pathologica 90(4): 383-387.

34. Guarner J, Brandt ME (2011) Histopathologic diagnosis of fungal infections in the 21st century. Clin Microbiol Rev 24(2): 247-280.
Marcelo V Radisic, et al. Extrapulmonary Pneumocystis Jirovecii Presenting as Fever and Lymphadenopathies. Case Report and Literature Review. J Inf Dis Trav Med 2018, 2(2): 000113. 
35. Huang YS, Yang JJ, Lee NY, Chen GJ, Ko WC, et al. (2017) Treatment of Pneumocystis Jirovecii pneumonia in HIV-infected patients: a review. Expert Rev Anti Infect Ther 15(9): 873-892.

36. Li H, Huang $H$, He H (2016) Successful treatment of severe Pneumocystis pneumonia in an immunosuppressed patient using caspofungin combined with clindamycin: a case report and literature review. BMC Pulm Med 16(1):144.
37. Tu GW, Ju MJ, Xu M, Rong RM, He YZ, et al. (2013) Combination of caspofungin and low-dose trimethoprim/sulfamethoxazole for the treatment of severe Pneumocystis Jirovecii pneumonia in renal transplant recipients. Nephrology (Carlton) 18(11): 736-742.

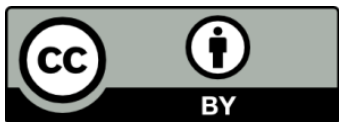

Marcelo V Radisic, et al. Extrapulmonary Pneumocystis Jirovecii 